\title{
L-Band Multiwavelength Erbium-Doped Fiber Ring Laser for Sensing Applications
}

\author{
R. A. Perez-Herrera ${ }^{a}$, A. Ullan ${ }^{b}$, D. Leandro ${ }^{a}$, M. Fernandez-Vallejo ${ }^{a}$, M.A. Quintela ${ }^{b}$, A. Loayssa ${ }^{a}$, \\ J. M. Lopez-Higuera ${ }^{b}$ and M. Lopez-Amo ${ }^{a}$ \\ ${ }^{a}$ Department of Electric and Electronic Engineering, Universidad Pública de Navarra, Campus \\ Arrosadía s/n E-31006 Pamplona, Spain. \\ ${ }^{\mathrm{b}}$ Photonics Engineering Group, University of Cantabria, Cantabria, Spain.
}

\begin{abstract}
In this work, a novel single-longitudinal-mode (SLM) four-wavelength laser configuration for sensing applications in L-band is proposed and experimentally demonstrated. This spectral region presents some advantages for the detection of dangerous gases. The sensor system presented here is based on ring resonators, and employs fiber Bragg gratings to select the operation wavelengths. The stable SLM operation is guaranteed when all the lasing channels present similar output powers. It is also experimentally demonstrated that when a SLM behavior is achieved, lower output power fluctuations are obtained.
\end{abstract}

Keywords: Erbium-doped fiber (EDF), fiber Bragg grating (FBG), multiwavelength lasing, optical fiber amplifier, optical fiber ring laser, single-longitudinal mode (SLM).

\section{INTRODUCTION}

Detection of trace gases such as methane, carbon monoxide and carbon dioxide is extremely important both for pollution monitoring and for safety reasons in the oil and gas industries, in water treatment plants, in landfill sites and in commercial or domestic environments, where methane gas may filter up through the ground and create an explosion hazard (the lower explosive limit, LEL, for methane is $5 \%$ by volume methane gas) [1]. Fiber optical sensors based on direct absorption spectroscopy, operating in the 1-2 $\mu \mathrm{m}$ spectral region, allow safe, remote location of sensors combined with the availability of low cost fiber components, connectors and compact gas cells. Several demonstrations of the application of the L-band hollow-core photonic bandgap fibers (HC-PBF) to the detection of methane have been reported in the literature [2]. It is therefore of commercial interest to develop portable gas sensors, based on optical techniques [3]. Optical sensors, based on semiconductor sources, have the potential advantages of: (i) intrinsically safe, (ii) ability to detect a specific gas by selection of appropriate wavelengths, (iii) able to operate in zero-oxygen environment (e.g. for purging of pipe lines), and (iv) low cost of maintenance, since the gas-detection principle is a physical process (not a chemical reaction), and therefore, poisoning of the sensor is not an issue (although dirt/contamination on the optics needs to be considered in the sensor design).

Regarding these optical techniques, it is worth noticing that stable single-longitudinal-mode (SLM) erbium-doped fiber lasers (EDFLs) have attracted great interest recently, because they can be potentially applied in fiber sensing systems, dense wavelength division multiplexed (DWDM) communications, high-resolution spectroscopy, and microwave photonics fields [4]. Several approaches have been employed to implement SLM operation in EDFLs, such as short linear cavity distributed Bragg reflector fiber lasers, fiber distributed feedback lasers, and fiber ring lasers [5] with different ring-cavity configurations. However, the fiber ring laser unavoidably generates an enormous number of densely spaced longitudinal modes lying beneath the erbium gain profile due to the required intracavity optical components, connecting fibers, and a rather long cavity length. Thus, fiber ring lasers usually perform unstably with a larger linewidth due to the multimode oscillation, mode competition, and hopping. To achieve SLM operation, a number of techniques have been proposed to ensure a long-term stable performance in EDFLs. Several previous studies proposed by the authors [5] show that, a single-mode operation of these laser systems in the C-band can be achieved when we emit simultaneously two wavelengths using a special ring cavity configuration. In this paper, we report, for the first time to our knowledge, a single-mode operation of an EDFL system operating in the L-band and for sensing applications; when we emit simultaneously four wavelengths using a specific ring cavity configuration. The stable SLM operation in this band is also guaranteed if the output power of all channels is similar. This implies that it is possible to avoid the utilization of additional optical filtering techniques (that reduce the optical efficiency) to achieve the SLM operation.

21st International Conference on Optical Fiber Sensors, edited by Wojtek J. Bock, Jacques Albert, Xiaoyi Bao, Proc. of SPIE Vol. 7753, 77533C · C 2011 SPIE · CCC code: 0277-786X/11/\$18 · doi: 10.1117/12.885129 


\section{EXPERIMENTAL SET UP}

The experimental setup of the proposed multiwavelength erbium-doped fiber ring laser (MEDFRL) is shown in Fig. 1. This set-up comprises an L-band EDFA module (EDFA-B-L-33 provided by MANLIGHT), four circulators, four FBGs in the L-band, four variable attenuators (VA) and one $4 \times 2$ optical coupler. This coupler is used for collecting the four arms signals and for extracting part of the optical output laser from the ring to the output port. The wavelength selection is carried out by means of FBGs and are centered at $1587.7 \mathrm{~nm}, 1591.9 \mathrm{~nm}, 1599.9 \mathrm{~nm}$ and $1610.2 \mathrm{~nm}$ with a corresponding full-width at half maximum (FWHM) of $0.17 \mathrm{~nm}, 0.18 \mathrm{~nm}, 0.27 \mathrm{~nm}$ and $0.08 \mathrm{~nm}$, respectively. In this topology, the FBGs reflected signals are inserted into the ring by using optical circulators conforming a serial topology and ensuring unidirectional operation and therefore, avoiding the spatial hole-burning (SHB) effect. Because of this, the use of isolators to ensure the unidirectional oscillation in this configuration was not necessary.

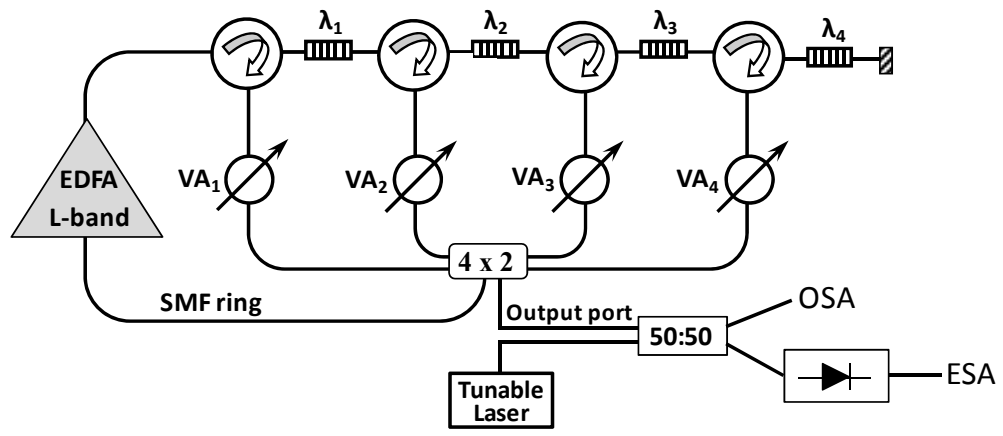

Fig. 1. Proposed single-longitudinal-mode four-wavelength fiber laser for sensors applications.

One of the major problems in multiwavelength ring lasers is that the oscillation threshold power for each wavelength is different due to the non-uniform shape of the EDF gain profile. As a consequence, variable attenuators (VA) have been connected to each FBG in order to correctly adjusting the cavity losses on each wavelength to achieve oscillation of the system in all the desired channels. All the free terminations have been immersed in refractive-index-matching gel to avoid undesired reflections. Several previous studies show that, in order to simplify this kind of setups, polarization maintaining (PM) components can be eliminated because of not having significant impact on the output power stability [6]. The laser output is monitored by an optical spectrum analyzer (OSA) with a highest spectral resolution of $0.01 \mathrm{~nm}$. Single-longitudinal-mode lasing is verified by heterodyne detection of the output signal. Each laser line is combined with the output of a commercial tunable laser source (TLS) with a linewidth of $100 \mathrm{KHz}$ closely located in the spectrum. In this way, the beating signal is observed by means of an ESA, whose resolution bandwidth can be as good as $1 \mathrm{~Hz}$.

\section{EXPERIMENTAL RESULTS AND DISCUSSIONS}

The output spectrum of the MEDFRL for a $23 \mathrm{dBm}$ pump power is shown in Fig. 2. As can be shown in this figure four lasing channels are obtained. The power of each of the four output channels is around $-27 \mathrm{dBm}$. For the four channels, the signal power is more than $50 \mathrm{~dB}$ higher than the amplified spontaneous emission (ASE) noise floor. Several previous studies show that these values are reasonably good for most sensor applications [7].

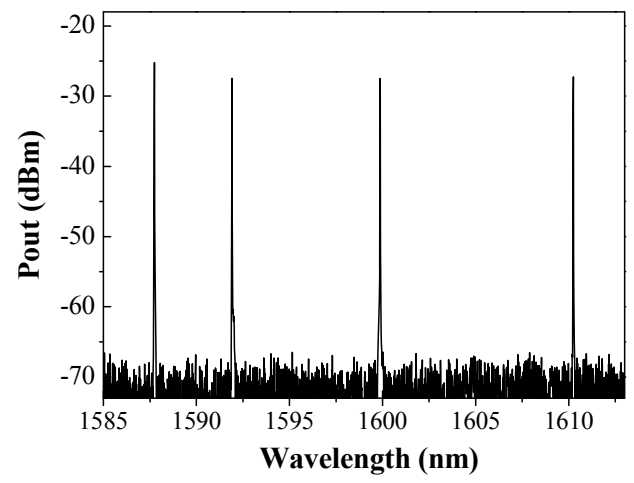

Fig. 2. Output optical spectrum for the MEDFRL circulators configuration with a pump power of $23 \mathrm{dBm}$. 
As it is well known, in this type of configurations, multiple longitudinal modes are supported by the cavity. However, as it will be exposed in this work, under some specific conditions, such as for similar pumping levels, a single-mode operation of the laser can be achieved. As reported in previous works by the authors [5], a SLM fiber ring laser can be made to annihilate the mode competition by using multiple lasing wavelengths. In this work, owing to the interaction of the seed light produced from one channel to the others and vice versa, multiple-longitudinal-mode oscillation can be suppressed, as can be seen in Fig. 3(a) and (b), and thus the mode competition and mode hopping is not produced. In these figures, the beat note spectrum between the tunable laser we used and the obtained single-longitudinal mode signal is presented. They show the obtained results when this tunable laser is fine tuned to the first (a) and second (b) wavelength laser emission in order to perform heterodyne detection.


Fig. 3. Output optical spectrum measured by the ESA for the MEDFRL circulators configuration when the tunable laser was tuned to the first (a) and second (b) wavelength laser emission.

In addition to this, authors would like to underline the fact that there are no peaks at low frequency except for a beat that is attributed to the residual side modes of the TLS. Thus, we can assure that these four wavelength lasers have a singlelongitudinal mode operation simultaneously. It is also worth noticing that the sidebands of the tunable laser can be also observed, as can be easily detect in Fig 3 (b), located very close to the beat peak. Due to the fact that only one peak is obtained for all the studied cases and taking into account that the resolution bandwidth of the ESA is good enough to discriminate two longitudinal modes separated about $8 \cdot 10^{-12} \mathrm{~nm}$, we can assure that this laser presents a SLM operation condition for all the channels at the same time. These measurements have been repeated at different emission wavelengths in the L-band. In all cases, an SLM operation for all the channels is achieved when all of these lasing wavelengths are oscillating simultaneously with similar output powers by using the VAs to adjust the cavity losses.

The utilization of this new laser for sensor multiplexing was carried out using the FBGs as temperature sensors. The temperature response of the sensors was tested by heating them on a climatic chamber in the range of $27^{\circ} \mathrm{C}$ to $75^{\circ} \mathrm{C}$. As can be seen in Fig 4, the center wavelength shift for the laser centered at $1610 \mathrm{~nm}$ presents a clear linear behavior (the mean square error is very close to 1) and a temperature sensitivity of about $10 \mathrm{pm} /{ }^{\circ} \mathrm{C}$ was measured for a threewavelength EDFRL.

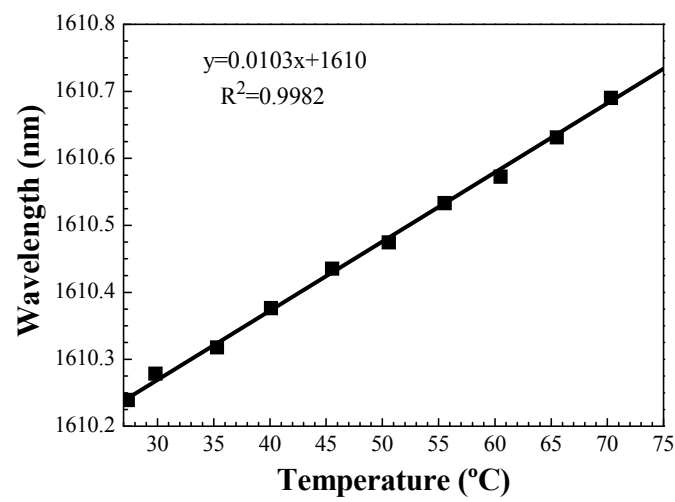

Fig. 4. Wavelength shift of one sensor against temperature for the three-wavelength EDFRL. 
The power of the laser output can suffer some changes with time. For this reason, the temporal variations of the output power were also measured and analyzed on this MEDFRL. The instability is defined as the output power for a given interval of time and a specific confidence level (CL). This is the probability value associated with a confidence interval (CI), given as a percentage. The CI is the estimated range of values where the parameter of interest is included [8].

It has been also experimentally demonstrated that when a SLM behavior is achieved, lower output power fluctuations are obtained. We have tested the sensor system during a period of 30 minutes. The measured data have been stored each $30 \mathrm{~s}$ and a CL of $90 \%$ was considered. Just to give data, for a dual-wavelength EDFRL at room temperature, the power averaged variation was around $2.1 \mathrm{~dB}$ for a multiple-longitudinal mode (MLM) behavior, and this value improves to be about $1.2 \mathrm{~dB}$ for a SLM operation.

Although we can conclude that a SLM operation implies also a remarkable improvement in terms of output power variations, the obtained results show that this configuration requires a more adequate control of the cavity losses on each wavelength to achieve oscillation of the system in all the desired channels and in order to obtain a very good equalized lasing system. This is an important study that we plan to perform because, in practice, it is not straightforward to achieve the optimum condition because of the availability of the optical variable attenuators and, therefore, it is relevant to know the level of performance degradation when, for example, the output power levels have great different values.

\section{CONCLUSIONS}

A novel single-longitudinal-mode four-wavelength laser configuration for sensing applications in L-band has been experimentally demonstrated in this work. This sensor system presented here is based on ring resonators, and employs fiber Bragg gratings to select the operation wavelengths. The stable SLM operation is guaranteed when all the lasing channels present similar output powers. It is also experimentally demonstrated that when a SLM behavior is achieved, lower output power fluctuations are obtained.

\section{ACKNOWLEDGEMENTS}

Financial support from the Spanish Comisión Interministerial de Ciencia y Tecnología within projects TEC200767987-C02-02 and TEC2010-20224-C02-01 is acknowledged.

\section{REFERENCES}

[1] Massie, C., Stewart, G., McGregor, G. and Gilchrist, J.R., "Design of a portable optical sensor for methane gas detection," Sensors and Actuators B 113(2), 830-836 (2006).

[2] Cubillas, A.M., Silva-Lopez, M., Lazaro, J.M., Conde, O.M., Petrovich, M.N., and Lopez-Higuera J.M., "Methane detection at 1670-nm band using a hollow-core photonic bandgap fiber and a multiline algorithm," Optics Express 15(26), 17570-17576 (2007).

[3] Mosely, P.T., Norris, J.O.W., and Williams, D.E., [Techniques and Mechanisms in Gas Sensing], Adam Hilger, Bristol, 234-259 (1991).

[4] Sun, J., and Huang L., "Single-longitudinal-mode fiber ring laser using internal lasing injection and self-injection feedback," Optical Engineering 46(7), 074201-6 (2007).

[5] Quintela, M.A., Perez-Herrera, R.A., Canales, I., Fernandez-Vallejo, M., Lopez-Amo, M., and Lopez-Higuera, J.M., "Stabilization of dual-wavelength erbium doped ring fiber lasers by single-mode operation", IEEE Photonics Technology Letters 22(6), 368-370 (2010).

[6] Perez-Herrera, R.A., Quintela, M.A., Fernandez-Vallejo, M., Quintela, A., Lopez-Amo M., and Lopez-Higuera, J.M., "Stability comparison of two ring resonator structures for multiwavelength fiber lasers using highly doped Erfibers", IEEE Journal of Lightwave Technology 27(14), 2563-2569 (2009).

[7] Diaz, S., Abad, S., and Lopez-Amo, M., "Fiber optic sensor active networking with distributed erbium doped fiber and Raman amplification," Laser \& Photonics Reviews 2(6), 480-497 (2008).

[8] http://www.stats.gla.ac.uk/steps/glossary/confidence_intervals.html 